\title{
Control of shivering with clonidine, butorphanol, and tramadol under spinal anesthesia: a comparative study
}

This article was published in the following Dove Press journal:

Local and Regional Anesthesia

9 August 201 I

Number of times this article has been viewed

\section{Pranav Bansal \\ Gaurav Jain}

Department of Anaesthesiology, Teerthanker Mahaveer Medical

College Moradabad, India
Correspondence: Pranav Bansal I35-Shanti Kutir, Manoranjan Park, Saket Road, Meerut Cantt, UP, India Tel +9l 9837009394

Email drpranav_bansal@yahoo.com
Introduction: The present study was designed to evaluate the efficacy of clonidine, butorphanol, and tramadol in control of shivering under spinal anesthesia, and to compare their side effects.

Methods: This randomized, prospective study was conducted in 90 patients who developed shivering under spinal anesthesia during various abdominal or orthopedic surgery procedures. On shivering, patients were randomly allocated to receive an intravenous, $1 \mathrm{~mL}$ bolus dose of $50 \mathrm{mg}$ tramadol, or $1 \mathrm{mg}$ butorphanol, or $150 \mu \mathrm{g}$ clonidine, in a double blinded manner. Control of shivering, time taken for cessation, recurrence, hemodynamic changes, axillary temperatures, and side effects were noted and compared for all 3 groups. Collected data were analyzed using appropriate statistical tests.

Results: Butorphanol and tramadol were more effective than clonidine in suppressing shivering. Butorphanol, tramadol, and clonidine completely controlled rigors in $83 \%, 73 \%$, and $53 \%$ of cases, respectively, and incompletely suppressed rigors in $16 \%, 26 \%$, and $46 \%$ of cases, respectively. Time taken to terminate rigors was significantly higher for clonidine ( $3.3 \pm 0.9$ minutes) than for butorphanol and tramadol ( $2.1 \pm 1.0$ minutes and $1.8 \pm 0.5$ minutes; $P<0.001)$.

Conclusion: Butorphanol had an edge over tramadol in controlling shivering with lower chances of recurrence, though both were superior to clonidine for this purpose with an early onset of action. We conclude that both these opioids control rigors better than $\alpha-2$ agonists.

Keywords: perioperative shivering, spinal anesthesia, tramadol, clonidine, butorphanol, thermoregulatory center

\section{Introduction}

Shivering, an involuntary, oscillatory muscular activity, is a physiological response to core hypothermia in an attempt to raise the metabolic heat production. ${ }^{1}$ Prolonged impairment of thermoregulatory autonomic control under anesthesia along with the cold environment of operating rooms and cold infusion fluids, contributes to a fall in core body temperature, and hence shivering. ${ }^{1,2}$ Other known causes of shivering include transfusion reactions, drug reactions, pre-existing high grade fever or bacteremia, or infusion of contaminated intravenous fluids (fungal growth in dextrose containing fluids). Perioperative hypothermia is the most common cause of shivering, though the exact incidence of each is difficult to evaluate. In a shivering patient, oxygen consumption may increase by $200 \%-500 \%$ along with a linear increase in carbon dioxide production. ${ }^{3}$ Thus in a patient with limited myocardial oxygen reserve or known coronary disease, shivering may further compromise myocardial function. ${ }^{4}$ Shivering also increases 
intraocular and intracranial pressure, and may contribute to increased wound pain, delayed wound healing, and delayed discharge from postanesthetic care. ${ }^{2}$ Apart from being an uncomfortable experience, its deleterious effects warrant primary prevention and prompt control on occurrence.

Various pharmacological therapies aim to prevent or treat shivering include opioids (pethidine, nalbuphine, or tramadol), ketanserin, propofol, granisetron, doxapram, physostigmine, clonidine, and nefopam, but debate on an 'ideal anti-shivering drug' continues. ${ }^{2,5}$ In a country like India, restrictions on drug licensing of opioids, and unavailability of many other drugs, compound the problem. ${ }^{6}$ Tramadol hydrochloride, a $\mu$-opioid receptor agonistic drug, has a modulatory effect on central mono-aminergic pathways, and thus inhibits the neuronal uptake of noradrenaline/serotonin and encourages hydroxytriptamine secretion which resets the body temperature regulation center. It has gained a reputation in many clinical trials for the control of shivering. ${ }^{6-8}$ Clonidine, an alpha-2 agonist drug, decreases the release of noradrenaline from the axonal terminals in the hypothalamus to exert its anti-shivering effects. ${ }^{9}$ Butorphanol, an easily available opioid, acts through $\mathrm{k}$ and $\mu$ receptor agonistic modulation, though only a few studies have denoted its antishivering properties. ${ }^{10}$ This clinical trial was set out to compare the efficacy of clonidine, butorphanol, and tramadol for controlling perioperative shivering of surgical patients under spinal anesthesia as a primary outcome. Secondary outcomes included perioperative variations in hemodynamic parameters and the incidence of adverse effects among the groups.

\section{Material and methods}

After obtaining institutional approval and written/informed consent from patients, we performed this randomized, double-blind study in cases of both genders between 18 and 65 years of age from July 2009 to January 2011 (1.5 years). Patients with American Society of Anesthesiologists physical status I-III, posted for procedures (urological, inguinal, and lower limb surgeries) under spinal anesthesia, who developed shivering during the intra or postoperative period (up to 2 hours) were included in the study. Patients with hypo or hyperthyroidism, morbid obesity (body mass index of $\geq 40 \mathrm{~kg} / \mathrm{m}^{2}$ ), fever (axillary temperature $>37^{\circ} \mathrm{C}$ ) and compromised cardiorespiratory functions were excluded.

A 2-operator technique was employed to maintain blinding. The cases were randomly allocated (sealed envelope technique) to one of 3 groups by an investigator intending to prepare the studied drug solution: Group $\mathrm{T}$ received an intravenous (iv) bolus of $50 \mathrm{mg}(1 \mathrm{~mL})$ tramadol
(Neon Labs, India); Group B received an iv bolus of $1 \mathrm{mg}$ (1 mL) butorphanol (Aristo Pharmaceuticals, India); Group $\mathrm{C}$ received an iv bolus of $150 \mu \mathrm{g}(1 \mathrm{~mL})$ clonidine (Neon Labs, India). Further interventions and monitoring were done by an investigator blinded to the group allocation.

In all cases, standard monitors were attached in the operating room and baseline parameters were recorded. Intravenous fluids infused were at room temperatures and ambient temperature of the operating room and recovery room was maintained at $22^{\circ} \mathrm{C}-28^{\circ} \mathrm{C}$. Spinal anesthesia was performed with a 25- or 26-gauge Quincke spinal needle, in a sitting position, at the $\mathrm{L}_{3-4 / 4-5}$ interspace (midline approach) with bupivacaine $(0.5 \%$, heavy) in a dose of $3.2-3.5 \mathrm{~mL}$, to achieve a desirable level at $\mathrm{T}_{8-10}$ dermatome, in accordance with the surgical procedure. After induction of spinal anesthesia, patients were observed for the occurrence of shivering until the postoperative period. The intensity of shivering was graded on a scale $0-3$ as: $0=$ no shivering; $1=$ shivering in face and head (mild), 2 = visible tremors involving more than one group of muscles (moderate), 3 = gross muscular activity involving the entire body, bed shaking (severe). Only cases that developed shivering of grade 2 or 3 during the perioperative phase were given treatment on an intention to treat basis. At the onset of shivering (grade 2 or 3 ), all patients were given oxygen via face mask at $6 \mathrm{~L} / \mathrm{min}$ and $1 \mathrm{~mL}$ of studied drug as per group allocation. Shivering control was defined as complete when post-treatment, the shivering score declined to 0 , incomplete when the scores decreased but did not abolish the shivering completely, and failed if no change in scores was observed. Sedation was assessed on a 4-point scale in which Grade $0=$ Alert; $1=$ Arouse to voice; $2=$ Arouse with gentle tactile stimulation; 3 = Arouse with vigorous tactile stimulation, and $4=$ No awareness.

The time taken for cessation of rigors and hemodynamic changes was recorded at regular 5-minute intervals up to 15 minutes. Recurring (any rise in shivering scores posttreatment) episodes of rigor and axillary temperatures at 2 data points (during shivering and 15 minutes post-treatment) were noted, and possible side effects of study drugs, in each group. Episodes of recurrence or incomplete control were treated with active warming measures using convection heaters, infusing moderately warm fluids or multimodal therapy (propofol [50 $\mu \mathrm{g} / \mathrm{kg}]$ and/or pethidine [0.5 mg/kg]) or both.

\section{Statistical analysis}

To detect a $20 \%$ difference in the response rate among the groups with a minimum response of $50 \%$ estimated from initial pilot observations, with $90 \%$ power and $5 \%$ alpha error (2-tailed), 
a sample size of 30 cases per group was required. Sample size was calculated using the power and sample size calculator of the Department of Biostatics, Vanderbilt University, US.

Statistical analysis was performed using SPSS 17.0 statistical software. The demographic characteristics, hemodynamics, axillary temperatures, duration of surgery, and time taken for cessation were noted and compared using one way ANOVA test. Post hoc intergroup comparisons were made using Bonferonni's correction. The ASA status and sedation score were noted and compared using the MannWhitney $U$ test. The nominal data including sex distribution, score, number of successful cessations, number of recurrences of shivering, and any side effects, were compared using Fisher's exact test/chi square test, whichever was appropriate. A $P$ value $<0.05$ was considered significant.

\section{Results}

In total, 530 spinal anesthetics were administered during the study period; 96 cases developed grade 2 and 3 shivering requiring treatment. Six cases were excluded from data analysis due to protocol violation, though all were given treatment. The study groups were comparable with no significant difference in demographic profile of cases, ASA health status, and duration of surgery (Table 1).

Though a decline in axillary temperatures was recorded at both the data points, no significant differences were observed between the groups. At the onset of shivering, the hemodynamic variables were comparable in all 3 groups. After receiving the blinded treatment, a propensity toward a fall in hemodynamic parameters was observed in all 3 groups with a significantly higher fall in systolic and diastolic blood pressure (Figures 1 and 2) and consequent rise in pulse rate values (Figure 3 ) in the clonidine group compared with the tramadol and butorphanol groups at various time intervals.

A significant decline in mean axillary temperatures $\left(1.2^{\circ} \mathrm{C}\right.$ to $1.4{ }^{\circ} \mathrm{C}$ ) was observed in all 3 groups during rigors and at post-treatment interval (after 15 minutes) compared with their baseline values (Figure 4).
The response rate (complete cessation of shivering after treatment) was $53 \%, 73 \%$, and $83 \%$ for Groups $\mathrm{C}$, T, and B, respectively, which was significantly low for patients treated with clonidine compared with tramadol and butorphanol (Table 2). The time taken for complete cessation of shivering was also significantly higher ( $3.3 \pm 0.9$ minutes $)$ in Group $C$ than in Group $\mathrm{T}(2.1 \pm 1.0$ minutes $)$ and $\mathrm{B}(1.8 \pm 0.5$ minutes $)(P<0.001)$, though the difference between tramadol and butorphanol was insignificant $(P=0.13)$. The degree of recurrent shivering was significantly less in butorphanol (2) treated cases as compared with those receiving clonidine (8) and tramadol (9). Five cases in Group T and 4 each in Group B and Group C (total 13) had nausea and vomiting at different time intervals, though the difference was non-significant in each group $(P>0.05)$. A comparatively higher incidence of grade 1 and 2 sedation was observed in butorphanol-treated cases (12/7) compared with Group T (3/0) and Group C (8/3) respectively. The incidence of grade 2 sedation was significantly higher $(P=0.023)$ in $26 \%$ (7) of butorphanoltreated cases, compared with $10 \%$ (3) in clonidine-treated cases and $0 \%$ in tramadol-treated cases. Episodes of oxygen desaturation or respiratory depression were not detected in any patient of any group during the study $(P>0.5)$, probably due to oxygen supplementation during shivering.

\section{Discussion}

Unfortunately, shivering presents as a common perioperative problem causing hypertension, tachycardia, and increased metabolic demands. It also interferes with intraoperative monitoring of electrocardiogram, blood pressure, and pulse oxygen saturation. ${ }^{5}$ Various risk factors associated with shivering include type and duration of anesthesia, level of sensory blockade, age, and temperature of the operating room and infusion fluids. ${ }^{11}$ Postoperative shivering occurred in 5\%-65\% of patients recovering from general anesthesia and in 30\% of volunteers undergoing regional anesthesia, though an extensive data search did not reveal any new study to support this observation. ${ }^{3}$ Shivering was observed to occur more frequently in the female gender and on use of thiopentone compared with

Table I Demographic profile of patients in all groups

\begin{tabular}{|c|c|c|c|c|}
\hline Patient variables & Group C & Group T & Group B & $P$-value \\
\hline Age (years) & $34 \pm 12$ & $33 \pm 14$ & $38 \pm 16$ & $0.91 *, 0.19 * *, 0.30^{\#}$ \\
\hline $\operatorname{Sex}(M / F)$ & $21 / 9$ & $25 / 5$ & $23 / 7$ & $0.22 *, 0.5 \mathrm{I}^{* *}, 0.55^{\#}$ \\
\hline Weight (kg) & $49 \pm 13.5$ & $54 \pm 15.8$ & $53 \pm 11.0$ & $0.18^{*}, 0.92^{* *}, 0.2 I^{\#}$ \\
\hline ASA status $(I / I I / I I I)$ & $21 / 7 / 2$ & $22 / 5 / 3$ & $25 / 2 / 3$ & $0.75^{*}, 0.47^{* *}, 0.18^{\#}$ \\
\hline Duration of Surgery (min) & $64 \pm 23$ & $72 \pm 27$ & $66 \pm 16$ & $0.22 *, 0.34 * *, 0.85^{\#}$ \\
\hline IV fluid infused $(\mathrm{mL})$ & $1103.82 \pm 125.9$ & $1 \mid 46.87 \pm 170.8$ & $1153.82 \pm 151.6$ & $0.39 *, 0.99 * *, 0.23^{\#}$ \\
\hline
\end{tabular}

Notes: *Group C vs T; **Group T vs B; \#Group C vs B.

Abbreviation: ASA, American Society of Anesthesiologists. 


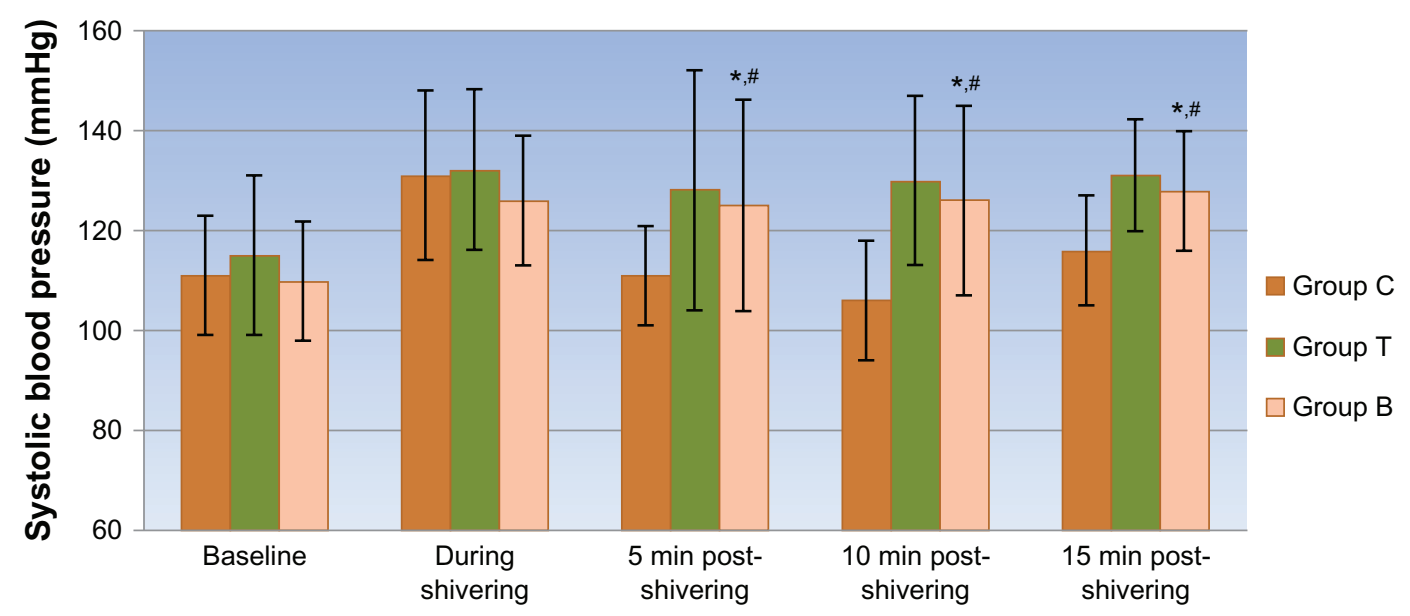

Time interval

Figure I Mean systolic blood pressure at different time intervals in all 3 groups.

Notes: *Group C vs T; **Group T vs B; ${ }^{*}$ Group C vs B: Symbols indicate points where $P<0.05$ on intergroup comparison.

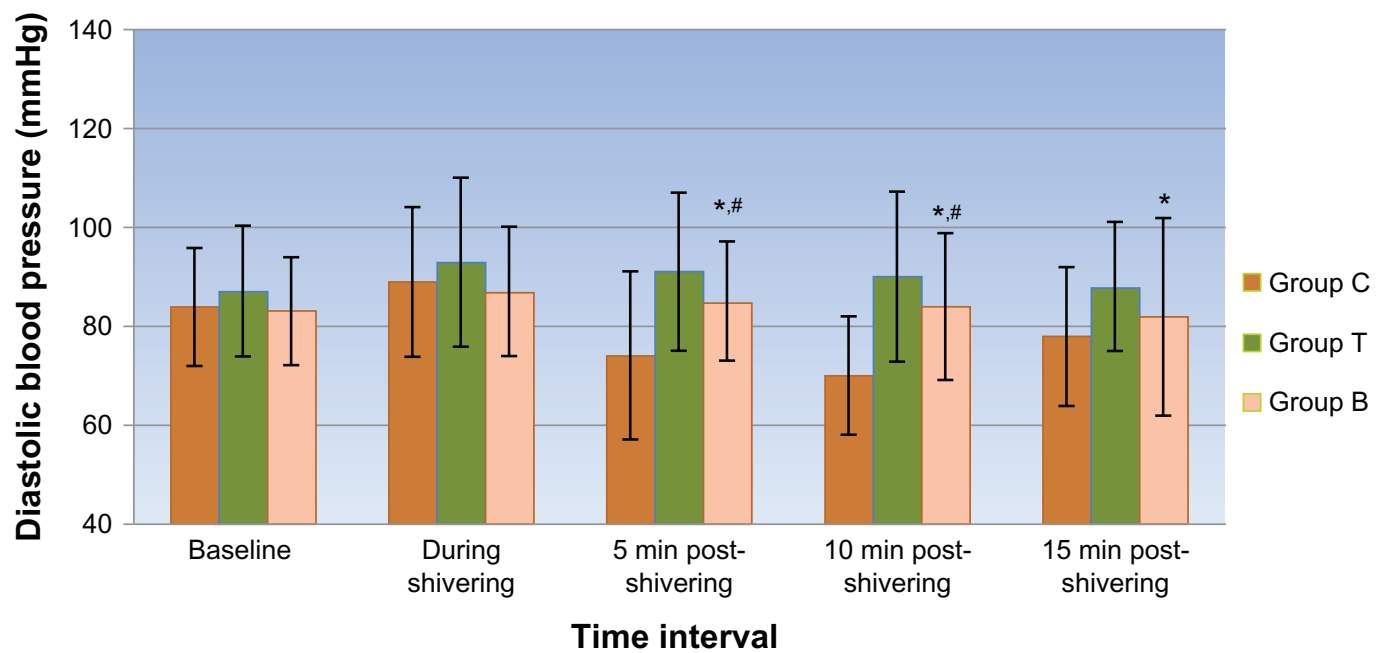

Figure 2 Mean diastolic blood pressure at different time intervals in all 3 groups.

Notes: *Group C vs T; **Group T vs B; "Group C vs B: Symbols indicate points where $P<0.05$ on intergroup comparison.

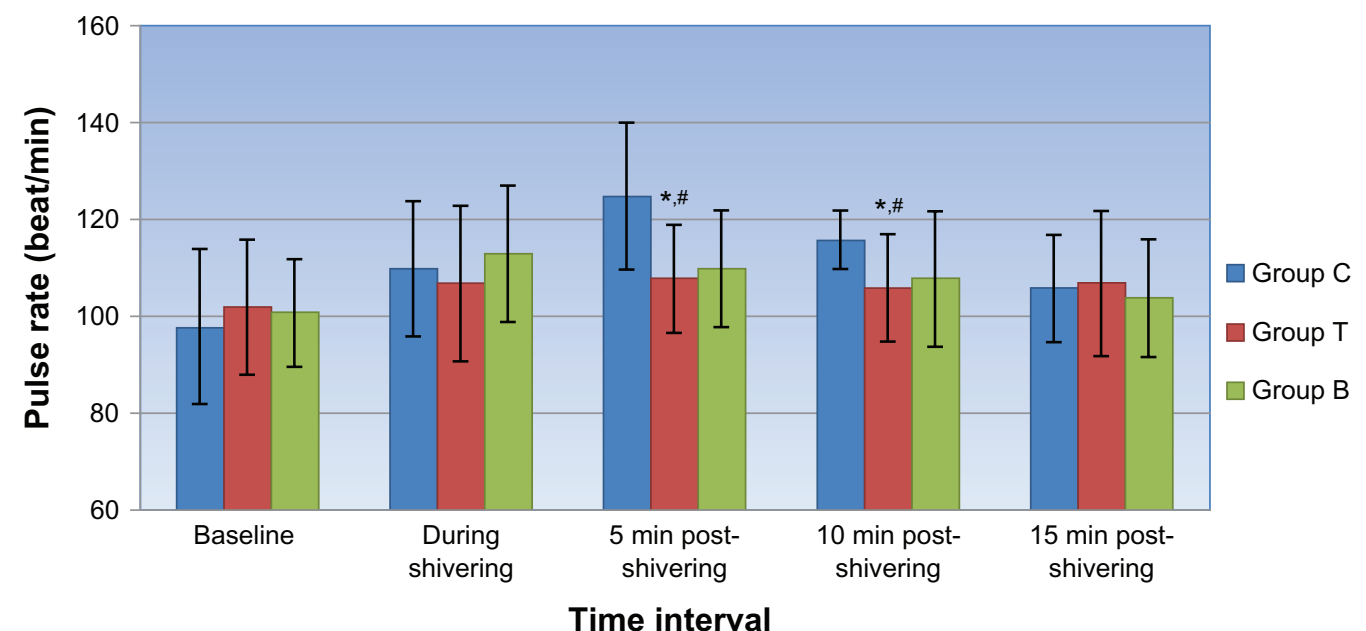

Figure 3 Mean pulse at different time intervals in all 3 groups.

Notes: *Group C vs T; **Group T vs B; ${ }^{*}$ Group C vs B: Symbols indicate points where $P<0.05$ on intergroup comparison. 


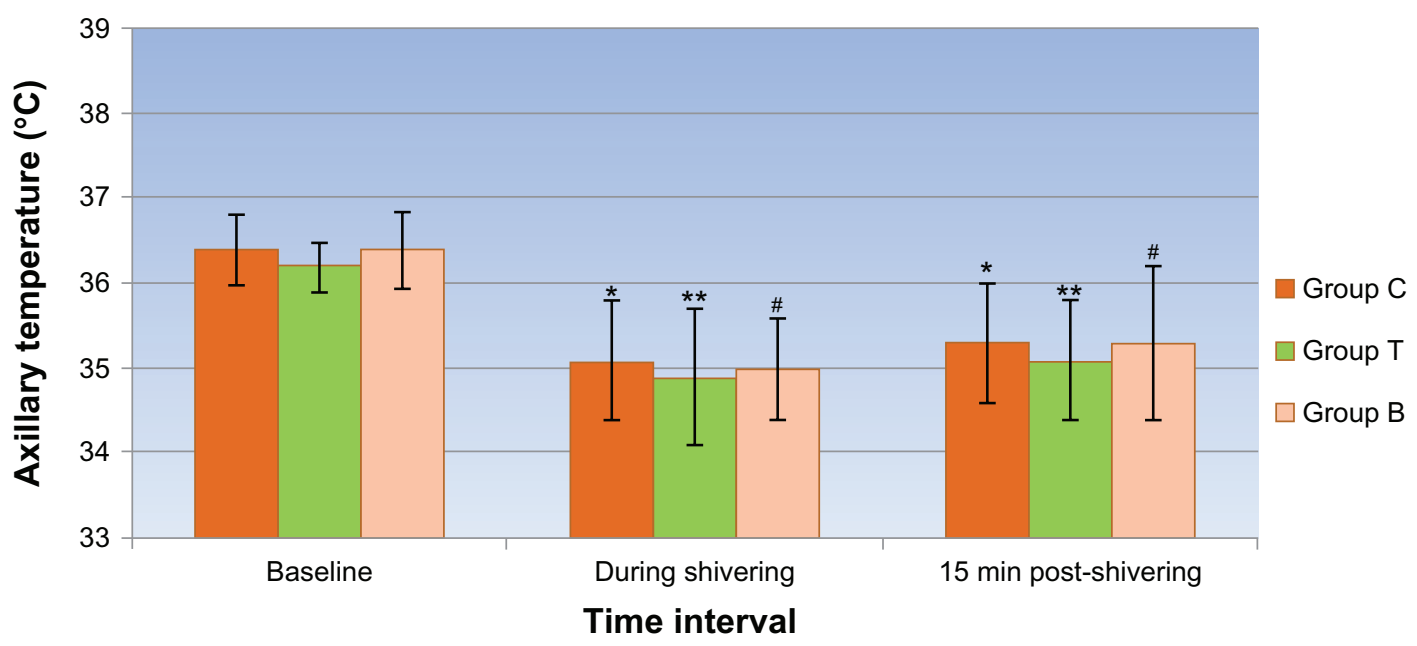

Figure 4 Mean axillary temperature at different time intervals in all 3 groups.

Notes: *Group C; **Group T; ${ }^{*}$ Group B: Symbols indicate points where $P<0.05$ on intra-group comparison compared with baseline values.

propofol after general anesthesia. ${ }^{12}$ Rigor occurs commonly, as a protective response to core hypothermia, though it may occur in the presence of normothermia. We also observed a significant fall in axillary temperatures $(P<0.001)$ in all 3 groups during shivering and post-treatment thereafter, compared with their baseline values. A non-significant temperature difference was observed when the data on cases during shivering and post-treatment were compared, which indicates that cessation of rigors was not related to modulation in body temperature, but was probably due to resetting of thermoreceptors at a lower threshold by the studied drugs.

Pharmacological intervention does not raise body temperature, but resets the shivering threshold to a lower level, thereby decreasing rigors and its episodes. The neurotransmitter pathways involved in shivering are complex and involve opioids, $\alpha-2$ adrenergic, serotenergic, and anticholinergic receptors. By virtue of this fact, drugs acting on these systems are utilized in treatment of this condition. ${ }^{1,2}$

Our results showed the superiority of butorphanol over tramadol and clonidine. Tramadol and butorphanol both had comparable results in complete suppression of shivering which accords with observations made by Atashkhoyi and Negargar, ${ }^{13}$
Dhimar et al, ${ }^{14}$ and Bhatnagar et al. ${ }^{15} \mathrm{~A}$ higher incidence of recurrence of rigors was observed in tramadol-treated patients in our study, which is in contrast to observations made by Maheshwari et $\mathrm{al}^{10}$ who observed a lower rate of recurrence with tramadol compared with butorphanol ( $8 \%$ vs $25 \%$ respectively). Clonidine-treated patients had a lower efficacy in control of shivering, with comparably higher recurrence than patients in the other 2 groups. This finding is in contradiction with the study done by Schwarzkopf et $\mathrm{al}^{16}$ and Horn et al ${ }^{17}$ who noted $100 \%$ response rates in control or prevention of rigors with clonidine after general anesthesia, though these authors also observed recurrences with clonidine. The time taken for control of shivering was also significantly higher with the clonidine group than with the tramadol and butorphanol groups. The time to control rigors was less than 5 minutes in studies done by most of the investigators using opioids, which proves the advantage of opioids in control of shivering compared with $\alpha-2$ agonists or other groups of drugs. ${ }^{7-10,14-16}$

The incidence of nausea and vomiting was comparable in all 3 groups with no statistical difference. Contrary to our results, previous studies have reported a higher incidence of nausea and vomiting in patients treated with tramadol

Table 2 Effect of studied drugs in all 3 groups and their significance

\begin{tabular}{|c|c|c|c|c|}
\hline Variable & Group C (\%) & Group T (\%) & Group B (\%) & $P$ value \\
\hline \multicolumn{5}{|l|}{ Shivering control } \\
\hline Complete & $16(53.3)$ & $22(73.3)$ & $25(83.3)$ & $0.10^{*}, 0.34^{* *}, 0.012^{\#}$ \\
\hline Incomplete & $7(46.6)$ & $5(26.6)$ & $4(16.6)$ & $0.5 I^{*}, 0.7 I^{* *}, 0.3 I^{\#}$ \\
\hline Time taken for cessation (min) & $3.3 \pm 0.9$ & $2.1 \pm 1.0$ & $1.8 \pm 0.5$ & $<0.00 I^{*}, 0.13^{* *},<0.00 \mathrm{I}^{\#}$ \\
\hline Recurrence of shivering & 8 & 9 & 2 & $0.77^{*}, 0.01^{* *}, 0.03^{\#}$ \\
\hline Nausea and vomiting & 4 & 5 & 4 & $0.7 I^{*}, 0.7 I^{* *}, I^{\#}$ \\
\hline Sedation score: I/2 & $8 / 3$ & $3 / 0$ & $12 / 7$ & $0.03^{*},<0.001^{* *}, 0.10^{\#}$ \\
\hline
\end{tabular}

Notes: *Group C vs T; **Group T vs B; *Group C vs B. 
compared with clonidine or butorphanol. Studies conducted by Gangopadhyay et a ${ }^{18}$ reported a higher incidence of vomiting with tramadol than pethidine, while Maheshwari et al ${ }^{10}$ showed a higher incidence of vomiting with butorphanol compared with tramadol. The literature supports a higher incidence of emesis with opioids, though the doses used by us were infrequently associated with this adverse effect.

It is also well known that repeated dosing of tramadol is associated with a high incidence of emesis, butorphanol with sedation and respiratory depression, and clonidine with hypotension and somnolence. The incidence of these side effects was higher in other studies after repeated drug dosing to abolish rigors, which may explain the reason for their lower incidence in our study. ${ }^{10,14,15,19}$

Pethidine is known to be the ideal drug for prevention and control of rigor under regional anesthesia or after recovery from general anesthesia, with $80 \%-85 \%$ success rates in various studies. We observed the superiority of butorphanol ( $83 \%$ cases) over tramadol (73\% cases) and clonidine $(63 \%$ cases) as an anti-shivering agent for the following reasons: an early onset of action; a higher rate of cessation of shivering; and lesser recurrence of shivering.

The limitations of our study include a relatively small sample size in proportion to the burden of this perioperative problem. The results of our study may not coincide with studies done on other ethnic populations owing to variations in body surface area and their heat or cold intolerance. Moreover, performing a dose-response study using a single drug could have delineated its anti-shivering profile and corresponding increase in side effects more profoundly. Future studies can investigate these aspects or compare the efficacy of a combination of drugs for prevention or control of rigors.

\section{Conclusion}

We conclude that butorphanol and tramadol are superior to clonidine for management of postoperative shivering due to higher rates of success, earlier onset of action and lesser recurrence with comparable levels of safety. Currently opioids hold a high reputation as reliable anti-shivering agents, though the search for an ideal substitute still continues.

Local and Regional Anesthesia

\section{Publish your work in this journal}

Local and Regional Anesthesia is an international, peer-reviewed, open access journal publishing on the development, pharmacology, delivery and targeting and clinical use of local and regional anesthetics and analgesics. The journal welcomes submitted papers covering original research, basic science, clinical studies, reviews \& evaluations,

\section{Disclosure}

The authors disclose no conflicts of interest.

\section{References}

1. De Witte J, Sessler DI. Perioperative shivering: physiology and pharmacology. Anaesthesiology. 2002;96:467-484.

2. Kranke P, Eberhart LH, Roewer N, Tramer MR. Pharmacological treatment of postoperative shivering: a quantitative systematic review of randomized controlled trials. Anesth Analg. 2002;94:453-460.

3. Dal D, Kose A, Honca M, Akinci SB, Basgul E, Aypar U. Efficacy of prophylactic ketamine in preventing postoperative shivering. $\mathrm{Br} J$ Anaesth. 2005;95:189-192.

4. Buggy DJ, Crossley AWA. Thermoregulation, mild perioperative hypothermia, and post-anaesthetic shivering. Br J Anaesth. 2000;84: 615-628.

5. Zhang Y, Wong KC. Anesthesia and postoperative shivering: its etiology, treatment and prevention. Acta Anaesthesiol Sin. 1999;37:115-120.

6. Katyal S, Tewari A, et al. Shivering: anesthetic considerations. J Anaesth Clin Pharmacol. 2002;18:363-376.

7. Bhatnagar S, Saxena A, Kannan TR, Punj J, Panigrahi M, Mishra S. Tramadol for postoperative shivering: a double blind comparison with pethidine. Anaesth Intensive Care. 2001;29:149-154.

8. Zahedi H. Comparison of tramadol and pethidine for postanesthetic shivering in elective cataract surgery. Journal of Research in Medical Sciences. 2004;5:235-239.

9. Piper SN, Maleck WH, Boldt J, Suttner SW, Schmidt CC, Reich DG. A comparison of urapidil, clonidine, meperidine and placebo in preventing postanesthetic shivering. Anesth Analg. 2000;90:954-957.

10. Maheshwari BS, Shah SK, Chadha IA. Tramadol and butrophanol for control of shivering: randomised double blind comparative study. J Anaesth Clin Pharmacol. 2008;24:343-346.

11. Sessler DI, Ponte J. Shivering during epidural anaesthesia. Anesthesiology. 1990;72:816-821.

12. Singh P, Harwood R, Cartwright DP, Crossley AWA. A comparison of thiopentone and propofol with respect to the incidence of postoperative shivering. Anaesthesia. 1994;49:996-998.

13. Atashkhoyi S, Negargar S. Effect of tramadol for prevention of shivering after spinal anaesthesia for cesarean section. Research Journal of Biological Sciences. 2008;3:1365-1369.

14. Dhimar AA, Patel MG, Swadian VN. Tramadol for control of shivering: comparison with Pethidine. Indian J Anaesthesia. 2007;51:28-31.

15. Bhatnagar S, Saxena A, Kannan TR, Punj J, Panigrahi M, Mishra S. Tramadol for postoperative shivering: a double-blind comparison with pethidine. Anaesth Intensive Care. 2001;29(2):149-154.

16. Schwarzkopf KR, Hoff H, Hartmann M, Fritz HG. A comparison between meperidine, clonidine and urapidil in the treatment of postanesthetic shivering. Anesth Analg. 2001;92:257-260.

17. Horn EP, Werner C, Sessler DI, Steinfath M, Esch JS. Late intraoperative clonidine administration prevents postanesthetic shivering after total intravenous or volatile anesthesia. Anesth Analg. 1997;84:613-617.

18. Gangopadhyay S, Gupta K, Acharjee S, Nayak SK, Dawn S, Piplai G. Ketamine, tramadol and pethidine in prophylaxis of shivering during spinal anaesthesia. J Anaesth Clin Pharmacol. 2010;26(1):59-63.

19. Dewitt J, Deloo FT, Deveylder J, Housmans PR. Tramadol in treatment of post anesthetic shivering. Acta Anesthesia Scand. 1997;41:506-510.

\section{Dovepress}

guidelines, expert opinion and commentary, case reports and extended reports. The manuscript management system is completely online and includes a very quick and fair peer-review system, which is all easy to use. Visit http://www.dovepress.com/testimonials.php to read real quotes from published authors. 